Bădescu Anca Gabriela, Tica C., Mihai Larisia

\title{
Congenital bilateral hydronephrosis diagnosed before birth, associated with vesicoureteral reflux, highlighted by urinary tract infection at the he age of 2 months - case presentation
}

Clinical County Emergency Hospital Constanța, Romania

\begin{abstract}
Vesicoureteral reflux is characterized by the retrograde flow of urine from the bladder to the kidneys. Vesicoureteral reflux may be associated with urinary tract infection, hydronephrosis, and abnormal kidney development (renal dysplasia). Hydronephrosis literally "water inside the kidney" - refers to distension and dilatation of the renal pelvis and calyces, usually caused by obstruction of the free flow of urine from the kidney. Untreated, it leads to progressive atrophy of the kidney. In cases of hydroureteronephrosis, there is distention of both the ureter and the renal pelvis and ureteres.

However, in the current era, hydronephrosis that is evident on fetal ultrasonography often heralds a ureteral abnormality. Hydronephrosis is defined as dilatation of the renal pelvis and/or calyces.

Vesicoureteral reflux may present before birth as prenatal hydronephrosis, an abnormal widening of the ureter or with a urinary tract infection or acute pyelonephritis.

The authors present a case of bilateral VUR of IVth grade associated with congenital hydronephrosis of IIIrd grade, diagnosed before birth with bilateral hydronephrosis, and taken into evidence at 2 months when he was first diagnosed with urinary tract infection. Positive diagnosis was facilitated by laboratory investigations (urine analysis, urine culture, voiding cystourethrography, static renal scintigraphy).
\end{abstract}

Keywords: urinary tract infection, vesicoureteral reflux, congenital hydronephrosis, voiding cystourethrography, static renal scintigraphy

\section{Anca Gabriela Bădescu}

Clinical County Emergency Hospital Constanța,

Blv. Tomis, No 145, Constanța

Phone: 072223880

e-mail: badescuanca2000@yahoo.com

\section{Introduction}

Vesicoureteral reflux (VUR) is an abnormal movement of urine from the bladder into ureters or kidneys. Urine normally travels from the kidneys via the ureters to the bladder. In vesicoureteral reflux the direction of urine flow is reversed (retrograde) [1].

Symptoms such as painful urination or renal colic/flank pain are not symptoms associated with vesicoureteral reflux. Newborns may be lethargic with faltering growth, while infants and young children typically present with pyrexia, dysuria, frequent urination, malodorous urine, but only when urinary tract infection is present as the initial presentation of VUR [2].

Hydronephrosis is defined as dilatation of the renal pelvis and/or calyces [3,4]. It may occur as a result of impairment of urine flow or the retrograde flow of urine. In Europe, the more objective method of measuring the anterior-posterior pelvic diameter in the transverse plane is employed6, combined with a still subjective description of the degree of calyceal dilatation and quality of the parenchyma [4].

In utero, hydronephrosis is defined as fetal renal pelvic diameter $>5 \mathrm{~mm}$ in the second trimester and $>7 \mathrm{~mm}$ in the third trimester [6,7]. Urine formation is thought to begin between the 5 th and 8 th weeks of gestation8. At 20 weeks, the fetus produces approximately $5 \mathrm{cc}$ of urine per hour and by the 40 th week this increases to $50 \mathrm{cc}$ per hour.

The fetal bladder can be observed to empty on ultrasound by the 15 th week. The bladder capacity which is approximately $10 \mathrm{cc}$ at 30 weeks increases 
to $50 \mathrm{cc}$ at term [6]. Antenatally diagnosed dilatation of the urinary tract can result from either impairment of urine flow or retrograde reflux of urine. Urine flow impairment can occur at any level in the urinary tract, and may affect one or both sides. Dilatation of the renal pelvis and calyces is the first anatomical response to impairment of urine flow and may lead to histological damage of the renal parenchyma and changes in renal function. Histological damage is related to the degree and level of urine flow impairment and its duration. In the past, the management of hydronephrosis was straight forward because children presented with symptoms, particularly infection or pain, which warranted operation. Long term natural history studies show that dilatation often improves or resolves spontaneously $[3,6]$; hydronephrosis is not synonymous with obstruction $[7,8]$.

Early diagnosis and vigilant monitoring of VUR are the cornerstones of management. Voiding cystourethrography (VCUG) or radionuclear cystourethrography (RNC) is used to confirm the diagnosis of VUR. A dimercaptosuccinic acid (DMSA) renal scan is used to evaluate for any renal abnormalities. Until the reflux resolves or the reflux is surgically treated, the patient should undergo monitoring with cystography (RNC or VCUG) every 12-24 months. Serial ultrasonography can also be performed to evaluate renal growth, especially in patients with a history of renal abnormalities such as size discrepancy or hydronephrosis [2,5].

The goal of treatment of vesicoureteral reflux is to minimize infections, as it is infections that cause renal scarring and not the vesicoureteral reflux.

Minimizing infections is primarily done by prophylactic antibiotics in newborns and infants who are not potty trained. Prophylactic antibiotics are prescribed to reduce the risk of bacterial infection of the bladder while reflux is present. Medical treatment entails low dose antibiotic prophylaxis until resolution of VUR occurs. Antibiotics are administered nightly at $1 / 3$ the normal therapeutic dose. A surgical approach is necessary in cases where a breakthrough infection results despite prophylaxis, or there is non-compliance with the prophylaxis. Similarly if the VUR is severe (Grade IV \& V), there are pyelonephritic changes or congenital abnormalities. Other reasons necessitating surgical intervention are failure of renal growth, formation of new scars, renal deterioration and VUR in girls approaching puberty [4].

There are three types of surgical procedure available for the treatment of VUR: endoscopic (STING/HIT procedures); laparoscopic; and open procedures (Cohen procedure, Leadbetter-Politano procedure) $[1,2,5]$.

Bladder management to ensure good lower urinary tract hygiene should be considered in children who have undergone toilet training [2].

\section{Gase presentation}

We will discuss a case of bilateral VUR of IVth grade associated with congenital hydronephrosis of IIIrd grade, diagnosed before birth with bilateral hydronephrosis, and taken into evidence at 2 months when he was first diagnosed with UTI. In the Pediatric Surgery Clinic of the Emergency County Hospital Constanta was hospitalized a male patient aged 1 year and 8 months, with no symptoms on admission of fever or micturition disorders, such as pollakiuria or dysuria.

From physiological personal history, our case is a baby born at 39 weeks by caesarean section on maternal request, with a weight at birth of $3600 \mathrm{~g}$, APGAR score $=9$, without suffering at birth.

From past medical pathological circumstances we discuss about a patient diagnosed before birth by ultrasound performed at 7 months of gestation to the mother with bilateral hydronephrosis.

In evolution, at the age of 2 months after birth, when he was taken in evidence in the Department of Pediatrics, he was diagnosed with UTI with E. coli, despite the correct antibiotic prophylactic treatment made after antibiogramme with Sumetrolim, the patient has experienced other episodes of Proteus UTI 3 times, and Klebsiella and E. coli 2 times.

At the age of 2 months was performed renal scintigraphy and retrograde cystography highlighting a bilateral VUR of IVth grade. Disease history indicates that the patient was diagnosed before birth 
with bilateral hydronephrosis, at the age of 2 months was first diagnosed with urinary tract infection with $\mathrm{E}$. coli and was introduced in the prophylactic treatment of urinary infections for prevention of relapses, after the correct treatment of acute flare. Given the high degree of reflux, IVth grade bilateral, with UTI recurrences in prophylactic antibiotic treatment administered correctly, the patient was hospitalized for surgical treatment by endoscopic injection with DEFLUX. On examination we found a child with normal length and ponderal development (waist = $81 \mathrm{~cm}$, weight $=11 \mathrm{~kg}$ ) with normal colored skin. At the urogenital apparatus examination, we found the presence of spontaneous micturitions, without any pathological changes.

Paraclinic laboratory investigations revealed the dynamics at admissions in hospital: hemoglobin $=12.3 \mathrm{~g} / \mathrm{dL}-12.5 \mathrm{~g} / \mathrm{dL}-12.4 \mathrm{~g} / \mathrm{dL}, \mathrm{WBC}=$ $14730 / \mathrm{mm}^{3}-12800 / \mathrm{mm}^{3}-33700 / \mathrm{mm}^{3}-9800 /$ $\mathrm{mm}^{3}$ with granulocyte predominance, $\mathrm{ESR}=19$ $\mathrm{mm} / \mathrm{h}-13 \mathrm{~mm} / \mathrm{h}-34 \mathrm{~mm} / \mathrm{h}-7 \mathrm{~mm} / \mathrm{h}, \mathrm{CRP}$ dynamic positive, negative at last hospitalization, blood urea $=0.24 \mathrm{~g} \%$ o and serum creatinine $=0$, $61 \mathrm{mg} / \mathrm{dL}$, normal in dynamics, urine analysis with very common leukocyte, positive nitrites, $\mathrm{pH} 5$, but normal at current hospitalization. Urine culture was sterile in the hospital. Initial imaging investigations highlighted

1. Plain abdominal ultrasound showed bilateral uretero-hydronephrosis of IIIrd grade,

2. Retrograde cystography showed bilateral vesicoureteral reflux of IVth grade at 2 months, and cystographies made in dynamics showed the same degree of VUR (Figures 1 and 2).

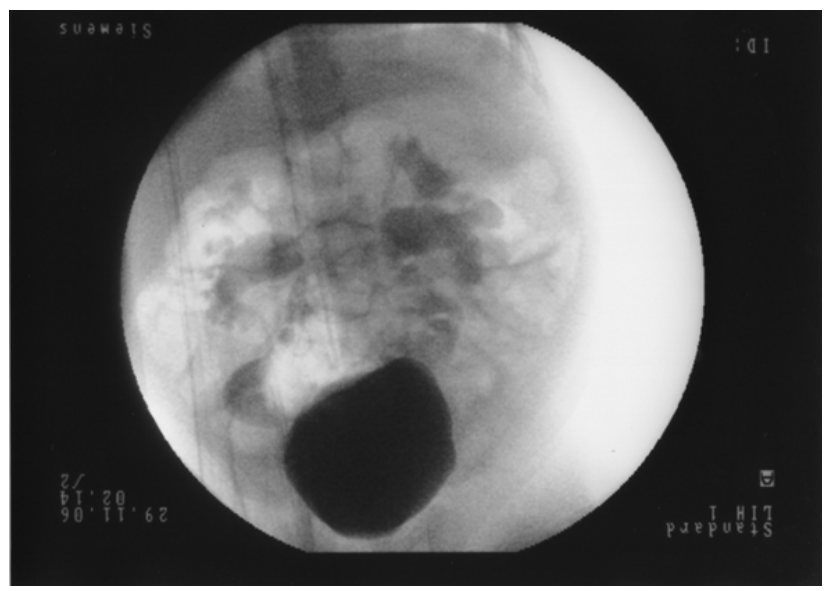

Figure 1 - Retrograde cystography performed at 2 months showed vesicoureteral reflux of IVth grade

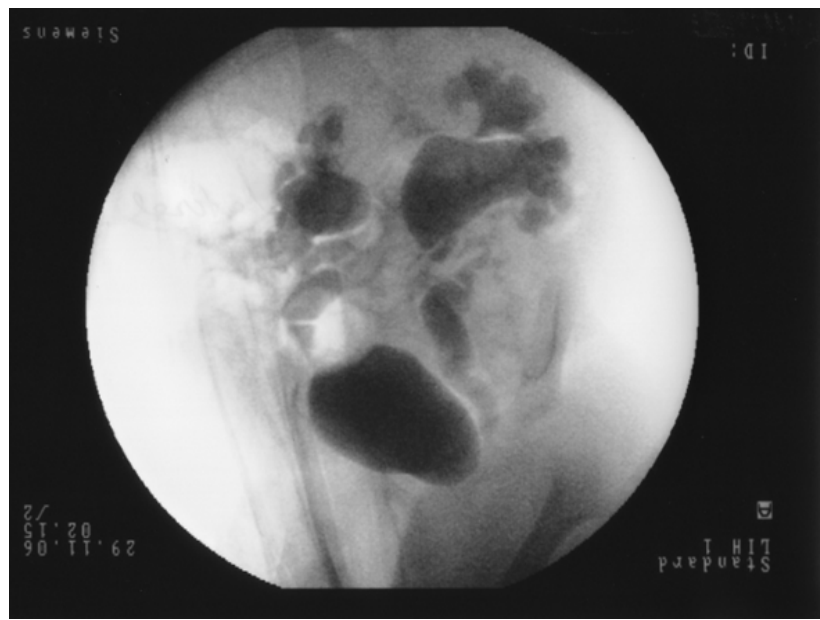

Figure 2 - Retrograde cystography performed at 2 months showed vesicoureteral reflux of IVth grade

3. Renal scintigraphy with Tc DTPA performed at 2 months showed right kidney infused (40.3\%), with a maximum of capture $(52.1 \%)$ at 16 minutes after injection, removing a small amount of radiotracer, the glomerular filtration curve being on the set. Middle lame has stasis. Small pyelocalyceal reflux episodes throughout the study. Right ureter dilated, with stasis from minute 12 to the end of the investigation. Infused left kidney $(59.7 \%)$, reaches a maximum of capture $(47.9 \%)$ at 27 minutes after injection. Slowly accumulation of tracer, glomerular filtration curve being upward. Small pyelocalyceal reflux episodes throughout the study. Left ureter has 

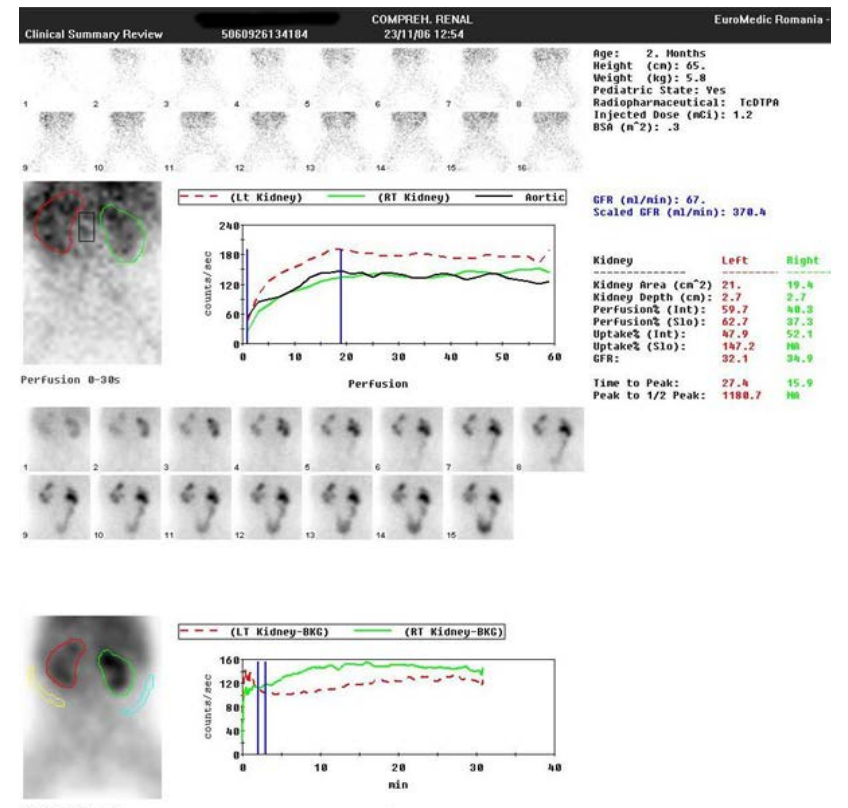

Figure 3 - Renal scintigraphy with Tc DTPA performed at 2 months-Glomerular filtration rate - $67 \mathrm{ml} / \mathrm{min}$

stasis in its lower third. Glomerular filtration $=67 \mathrm{ml}$ $/$ min (right kidney $=34.9 \mathrm{ml} /$ minute, left kidney $=$ $32.1 \mathrm{ml} /$ minute) (Figure 3).

It has been decided the association of cystoscopy surgery, of submucosal injection with DEFLUX. It was performed cystoscopy, which showed ureteric ectopic layers in form of "soccer stadium ", during which endoscopic treatment was performed of VUR by submucosal injection of DEFLUX - 2 ampoules, and urethrorectal-bladder catheter for 24 hours. The post-surgery evolution was favorable under afebrile conditions, suppression of Foley probe being performed at 48 hours postoperatively. It was recommended further prophylactic treatment postsurgery with Sumetrolim, one single dose in the evening.

At 3 days after surgery was performed ultrasound for control of substance injected and were observed post-surgery injection nodules (Figures 4 and 5).

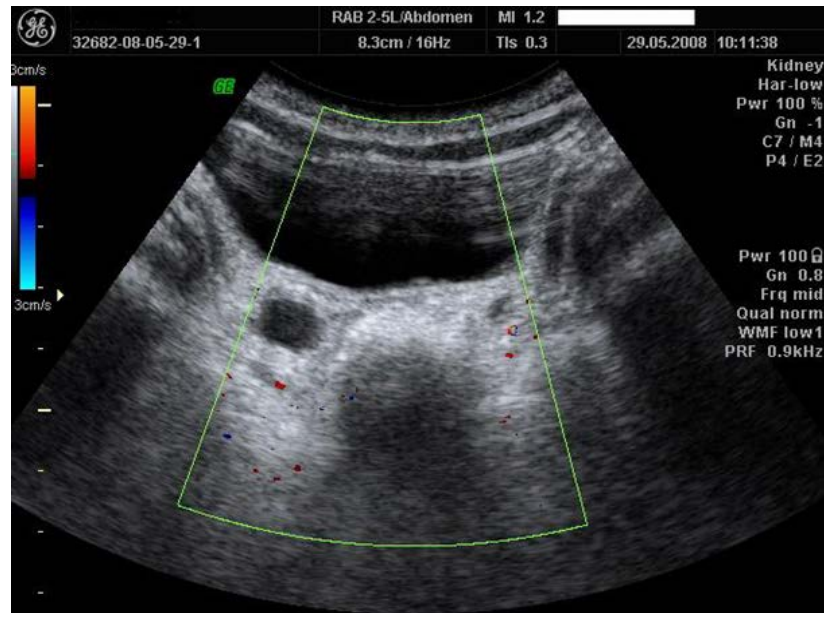

Figure 4 - post-surgery injection nodules

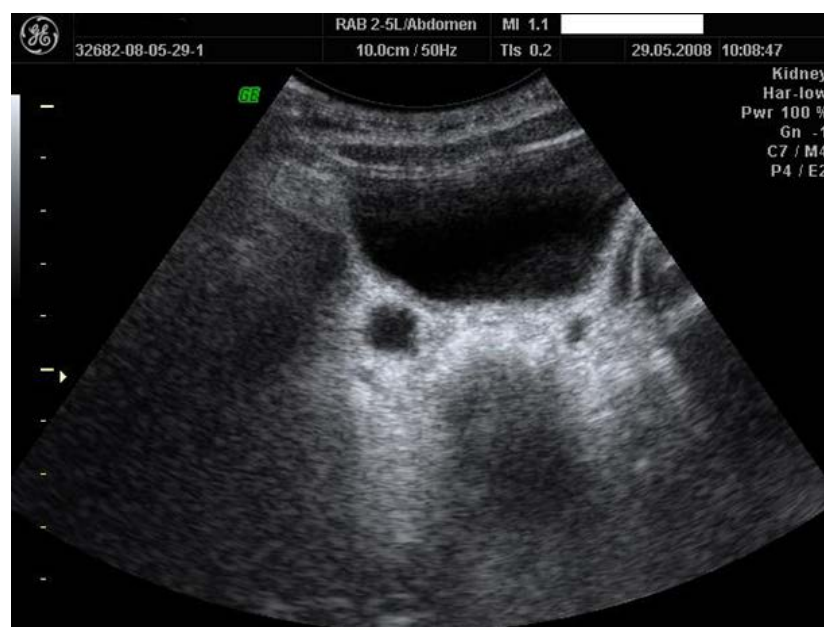

Figure 5 - post-surgery injection nodules

At 3 months after surgery was performed retrograde cystography of control which showed the disappearance of right VUR and left VUR reduction in IIIrd grade, and scintigraphy which showed the improvement of renal glomerular filtration (Figure 6). 


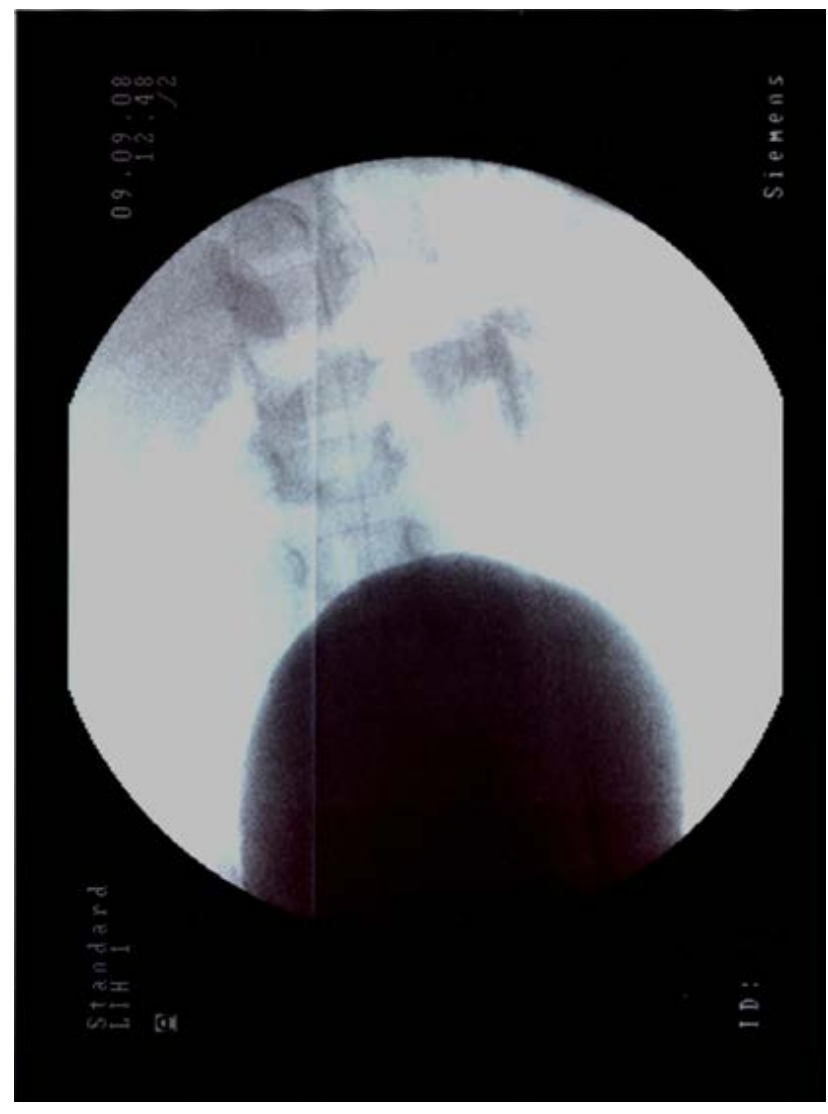

Figure 6 - Retrograde cystography at 3 months after surgery showed the disappearance of right VUR and left $V U R$ reduction in IIIrd grade

The renal control scintigraphy, performed 3 months after surgery with TcDTPA, showed the right kidney infused (49.3\%), with a maximum of capture $(67.8 \%)$ at 21 minutes after injection, glomerular filtration curve being upward. Multiple episodes of pyelocalyceal reflux. Stasis in pelvis remained until the end of investigation. Dilated right ureter, presenting stasis beginning at 10 minutes and until the end of investigation. Left kidney infused $(50.7 \%)$, with a maximum of capture $(32.2 \%)$ at 23 minutes after injection. Slow accumulating of tracer, glomerular filtration curve being upward. At the end of the investigation retain in parenchyma one small amount of tracer. Left ureter not being seen. Glomerular filtration $\mathrm{A}=85.1 \mathrm{ml} /$ minute (right kidney $=57.7 \mathrm{ml} /$ minute, left kidney $=27.4 \mathrm{ml} /$ min) (Figure 7).

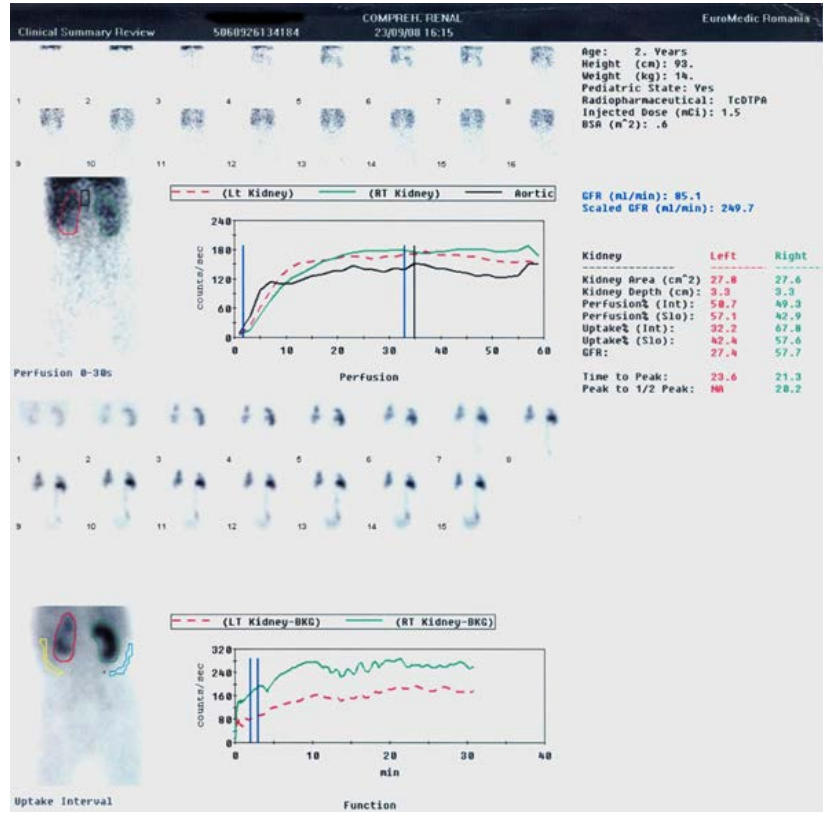

Figure 7 - Renal scintigraphy with Tc DTPA performed at 3 months after surgery (at 2 years age) - Glomerular filtration rate $-85,1 \mathrm{ml} / \mathrm{min}$

The patient was introduced in UTI relapse prevention program, immediately, by taking Zinat, $125 \mathrm{mg} / 5 \mathrm{ml}$ suspension, $2.5 \mathrm{ml} /$ day in the evening, 10 days / month, Negram $1 / 4$ tablet / day in the evening 10 days / month, Sumetrolim $25 \mathrm{mg}$ Trimetoprim / $5 \mathrm{ml}, 3.5 \mathrm{ml} /$ day in the evening 10 days / month, monthly monitoring of urine cultures, and clinical and paraclinical laboratory monitoring every 3 months. During this period the patient presented only two in hospital, the balance made showing 2 episodes of UTI, one with Proteus and one with E. coli, properly treated, 14 days, with Cefort 3 days $1 \mathrm{~g} /$ day, in Department of Pediatrics, and Cedax suspension $3 \mathrm{ml}$ / day 11 days at home, and ultrasound changes were stationary

Subsequently was carried associated phimosis treatment with continuing the prophylactic antibiotic treatment with Sumetrolim $5 \mathrm{ml} /$ day in the evening for 1 more year with monthly monitoring of urine cultures, and three months clinical and paraclinical monitoring in the laboratory. No new UTI episodes have been recorded. For technical reasons could not been made other cystography of control until now.

Abdominal ultrasound performed showed 
reduction in dynamic of uretero-hydronephrosis, last ultrasound performed in May 2012 showing a right kidney $=9,3 / 3,6 \mathrm{~cm}$, with hydronephrosis of Ist grade (anteroposterior diameter of the pelvis - 10.8 $\mathrm{mm}$ ), and a left kidney $=8,2 / 3 \mathrm{~cm}$, hydronephrosis of Ist (anteroposterior diameter of the pelvis - 13 $\mathrm{mm}$ ) (Figures 8 and 9).

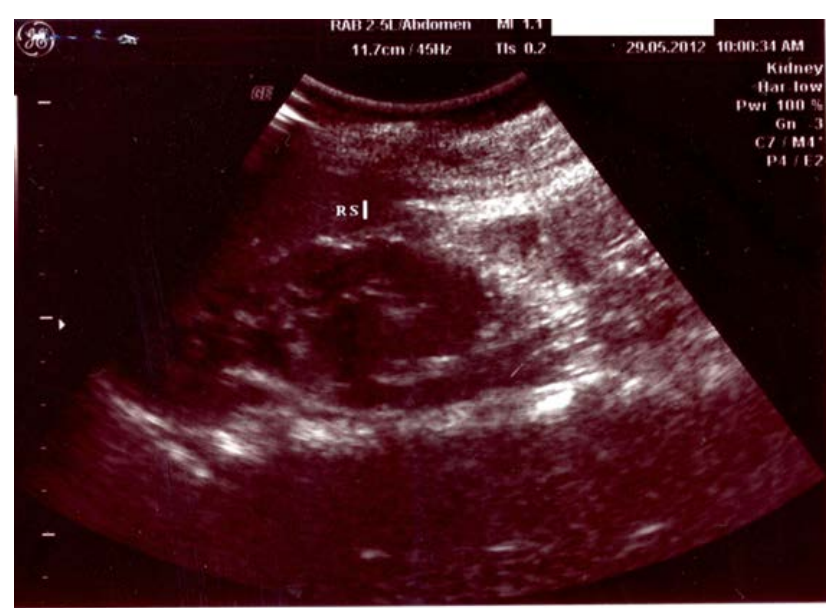

Figure 8 - Left Kidney -Abdominal ultrasound which shows bilateral hydronephrosis of Ist grade, 4 years after surgery with antibiotic prophylactic treatment administered correctly 2 years after surgery

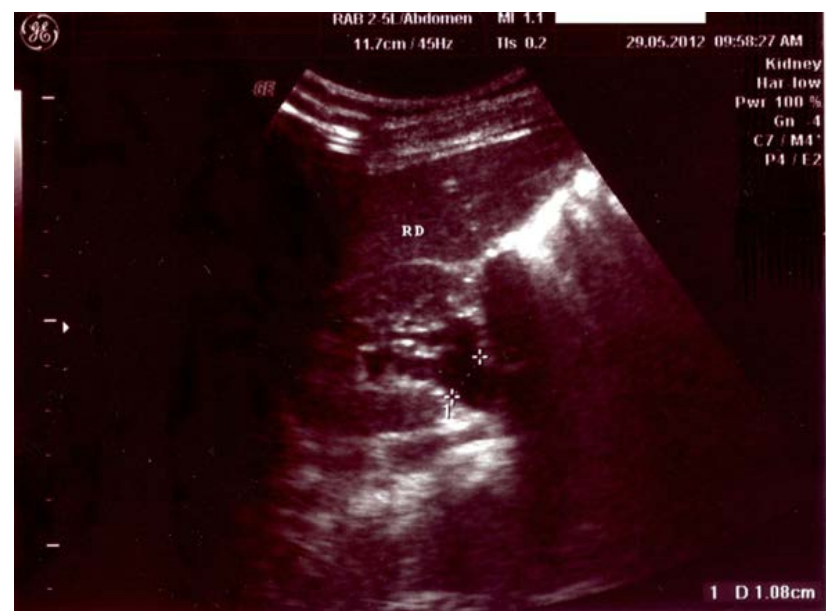

Figure 9 - Right Kidney - Abdominal ultrasound which shows bilateral hydronephrosis of Ist grade, 4 years after surgery with antibiotic prophylactic treatment administered correctly 2 years after surgery

Still being monitored, the patient aged 6 years, normally developed in length and weight, who has not experienced any episode of UTI during the last 2 years, makes at 3 months a clinical balance, with paraclinical tests (urine analysis, urine culture), and abdominal ultrasound every 6 months, being scheduled to repeat the cystography.

\section{Discussions}

This paper presents a special case of pediatric pathology, (gynecology, nephrology, urology), why? Because there has been implemented a protocol to establish pediatric urinary malformations, and implicitly, of the vesicoureteral reflux.

Positive diagnosis was facilitated by performing antenatal ultrasound, voiding cystourethrography and static scintigraphy TcDTPA [1,2].

Renal scintigraphy with Tc DTPA performed at 2 months showed low glomerular filtration rate and raised the need for emergency surgery to correct VUR. In this situation was performed endoscopic injection with DEFLUX (STING procedure) associated with recurrence prophylaxis with antibiotics.

Recurrence prevention of urinary tract infections is important in order to prevent appearance of new renal scars, and therefore introducing a long term antibiotic treatment scheme to the child, one evening, 1/3 of the dose, for 6 months, with further evaluation.

\section{Conclusion}

We tried to present a case of urinary-renal malformation, congenital bilateral hydronephrosis diagnosed before birth, associated with vesicoureteral reflux, manifested by urinary tract infection at 2 months age. 
This paper presented endoscopic injection with DEFLUX for a patient with this renal malformation which led to remission VUR with recurrence prophylaxis with antibiotics.

Positive diagnosis was facilitated by laboratory investigations (urine analysis, urine culture, voiding cystourethrography, static renal scintigraphy).

An early introduction of a protocol is important for early diagnosis of vesicoureteral reflux in order to maintain a normal renal function of the future adult.
Long-term followup of prenatally detected severe bilateral newborn hydronephrosis initially managed nonoperatively. J Urol. 168, 118-20.

8. Gordon, I., Dhillon, H.K., Gatanash, H. \& Peters, A.M. (1991). Antenatal diagnosis of pelvic hydronephrosis: assessment of renal function and drainage as a guide to management. $J \mathrm{Nucl}$ Med. 32, 1649-54

\section{References}

1. Peters, C.A. Skoog, S.J., Arant, B.S. Jr, Copp, H.L., Elder, J.S., Hudson, R.G., Khoury, A.E., Lorenzo, A.J., Pohl, H.G., Shapiro, E., Snodgrass, W.T. \& Diaz, M. (2010). Summary of the AUA Guideline on Management of Primary Vesicoureteral Reflux in Children. The Journal of Urology. 184(3), 1134-44.

2. Tekgül, S., Riedmiller, H., Hoebeke, P., Kočvara, R., Nijman, R.J., Radmayr, C., Stein, R. \& Dogan, H.S. (2012). European Association of, Urology "EAU guidelines on vesicoureteral reflux in children." European urology. 62(3), 534-42.

3. Kumar, V., Fausto, N., Robbins, S.L., Abbas, A.K. \& Cotran, R.S. (2005). Robbins and Cotran Pathologic Basis of Disease (7th ed.). Philadelphia PA: Elsevier Saunders. p. 1012-4.

4. Dhillon, H.K. (1998). Prenatally diagnosed hydronephrosis: the Great Ormond Street experience. Br J Urol. 81 Suppl 2, 39-44

5. Chiriac-Babei, C. \& Dima B.A. (2009). Importanța diagnosticului imagistic in refluxul vezicoureteral la copil, Revista română de pediatrie. $\operatorname{LVIII}(1), 40-45$

6. Koff, S.A. \& Campbell, K.D. (1994). The nonoperative management of unilateral neonatal hydronephrosis: natural history of poorly functioning kidneys. $J$ Urol. 152, 593-5.

7. Onen, A., Jayanthi, V.R. \& Koff, S.A. (2002). 